Revista

Actualidades Investigativas

en Educación

\title{
Percepción del profesorado y el alumnado universitario de la formación temprana en investigación científica
}

Perception of university teachers and students about early training in scientific research

pp. 1-20

Gladys del Carmen Medina Morales

\section{Citar este documento según modelo APA}

Medina Morales, Gladys del Carmen. (2020). Percepción del profesorado y alumnado universitario de la formación temprana en investigación científica. Revista Actualidades Investigativas en Educación, 20(3), 1-20. Doi. 10.15517/aie.v20i3.43674 


\title{
Percepción del profesorado y el alumnado universitario de la formación temprana en investigación científica
}

Perception of university teachers and students about early training in scientific research

\section{Gladys del Carmen Medina Morales ${ }^{1}$}

Resumen: En este artículo se presentan resultados del programa institucional en la Universidad Juárez Autónoma de Tabasco, (UJAT) en México, denominado Verano de la Investigación Científica (VIC), iniciativa que fue creada por la Academia Mexicana de Ciencias. El estudio tuvo como propósito identificar la percepción que el alumnado participante y el profesorado responsable tienen un específico en el programa, y a partir de ahí, se enriquecen las estrategias y mecanismos del VIC. Se trata de una investigación cuantitativa, descriptiva donde se aplicó una encuesta como instrumento de recolección de datos. La encuesta estuvo conformada por 9 ítems para el profesorado de ciencia y 6 para la población estudiantil. La participación fue de 1581 docentes $(H=851 \mathrm{M=730})$ y de 3046 estudiantes $(H=1194 \mathrm{M}=1852)$ de las 12 divisiones académicas, que participaron en el programa de Verano de la Investigación Científica del 2016 al 2020. Los resultados arrojaron una evaluación positiva del programa, del rol de participantes en general, así como la participación preponderante de mujeres. Se concluyó hacer una propuesta a las instancias correspondientes para crear un perfil del profesorado, reformular metodologías, instrumentos de evaluación que permitan mejorar la trazabilidad de la formación científica que se les imparte a la población estudiantil en dicha institución.

Palabras clave: formación, rol del profesorado, investigación científica, población estudiantil, educación superior.

\begin{abstract}
Results of the institutional program are presented at the Universidad Juárez Autónoma de Tabasco (UJAT), called Research Summer Program. The purpose of the study was to identify the perception that students and researchers had about themselves and each other as collaborators in the program and from there, enrich their strategies and mechanisms. The study design was descriptive where a survey was applied as an instrument for data collection. The survey consisted of 9 items for the researcher and 6 for the students. The participants were 1581 researchers $(M=851 W=730)$ and 3,046 students $(M=1194 W=1852)$ from the 12 academic divisions, who participated in the Research Summer Program from 2016 to 2020. The results showed a positive evaluation of the program, the role of researcher in general, as well as women's preponderant participation, however, it was concluded to make a proposal to the corresponding instances to create a profile of the researcher, as well as to reformulate methodologies, evaluation instruments that yield data that allow to improve the traceability of the scientific training that is offered to students in that institution.
\end{abstract}

Keywords: formation, teacher rol, scientist research, student population, higher education.

\footnotetext{
1 Profesora Investigadora de la Universidad Juárez Autónoma de Tabasco, de la División Académica de Educación y Artes; Tabasco, México. Doctora en Educación, Universidad del Sur, México. Orcid.: https://orcid.org/0000-0003$\underline{2238-1820}$
}

Dirección electrónica: mmg740731@hotmail.com

Artículo recibido: 31 de marzo, 2020

Enviado a corrección: 11 de agosto, 2020

Aprobado: 28 de agosto, 2020 


\section{Introducción}

El papel de las universidades ha ido cambiando con el paso de los años; actualmente son dinámicas, multidisciplinarias y diversificadas, una de las funciones sustantivas de las universidades es la investigación, y el profesorado que investiga debe contribuir en la generación del conocimiento y en la formación de grupos que persigan el desarrollo de habilidades investigativas, así como para revisar el estado del arte en su disciplina, instruir a otros y a partir de ahí, generar nuevos conocimientos. Como Quintero-Corzo dice que:

Los procesos formativos en la universidad han adoptado un supuesto básico: el educador es el primer actor capaz de construir saber pedagógico al comprender su acción, reflexionarla, experimentarla, escribirla y volver a reiniciar el proceso en sucesivos ciclos en su relación con el estudiante, el conocimiento, la cultura escolar, los procesos formativos, la ciencia y la tecnología. (2008, p. 32)

Uno de los inconvenientes presentados en el desarrollo de la investigación es la formación científica tardía en el nivel de pregrado, su acercamiento se da en niveles avanzados de la carrera, cuando deben decidir su modalidad de titulación. El profesorado que investiga, es quien "plantea situaciones problemáticas, a veces ni siquiera problemas acabados o bien estructurados, dejando que su estructuración sea parte del trabajo del estudiante." (Restrepo, 2002, p. 6). Lo anterior significa un riesgo, ya que pueden sentirse en desventaja cuando realizan estancias de investigación, y de esta manera caer en la desmotivación, significando un tropiezo.

Para Naranjo (2009), esta actividad es como una trama que sostiene el desarrollo de aquellas actividades que son significativas para la persona y en las que ésta toma parte. (p.153). Formar al estudiantado universitario en el ámbito científico es una tarea que el profesorado científico tiene entre sus funciones, en México hasta la década de los noventa se vio la importancia de crear un programa que aglutinara al estudiantado interesado en el campo científico, fue así como en 1991 el Verano de la Investigación Científica (VIC), fue creado por la Academia Mexicana de Ciencias (AMC) junto con diversas instituciones de Educación Superior del país y ha privilegiado la participación de estudiantes interesados en hacer investigación de todas las áreas de conocimiento en todo el país.

Desde su inicio, la AMC ha promovido la realización de estancias de investigación de jóvenes a nivel universitario que, "tienen como objetivo principal fomentar el interés de los estudiantes de licenciatura por la actividad científica en cualquiera de las áreas, a través de 
la realización de actividades propias de la investigación" (AMC, 2020, p. 1). De este modo, el estudiantado se siente acompañado, supervisado y guiado por el profesorado.

Este programa privilegia la formación científica en el nivel de pregrado, como Palés y Arcadi (2004) señalan que el Verano de Investigación Científica se constituye como un verdadero laboratorio de prácticas en un contexto real. Significa entonces que el VIC es un semillero en donde los procesos formativos en ciencia de las universidades germinan con la intervención del profesorado, quien debe ser una persona con experiencia y con disposición para dedicar una parte del tiempo de su actividad académica hacia la investigación para generar conocimiento y segundo, formar al estudiantado, de lo contrario el proceso se vería interrumpido por falta de experiencia de quien forma.

En el contexto local, la Universidad Juárez Autónoma de Tabasco (UJAT), en donde se desarrolla esta investigación, desde su Plan de Desarrollo Institucional 2004-2008, trazaba una línea estratégica para "diseñar y desarrollar un programa de investigación temprana para incorporar a estudiantes de las diferentes carreras a proyectos de investigación realizados por los académicos de la universidad, con el objetivo de formación en la investigación" (UJAT, 2004, p. 74).

Desde entonces, la UJAT ha asignado un techo financiero al programa y es así como el VIC quedó inserto en los Planes de Desarrollo Institucional vigentes. Esto significó una mayor cobertura, en todas las divisiones académicas contaron con la difusión necesaria para postularse y ser parte de esta actividad. La UJAT desde su inicio, participó en la actividad de fomento a la formación científica, la cual se fue incrementando tal como se muestra en la Figura 1. Como se mencionó con anterioridad, el programa adquirió relevancia como generador de la formación científica entre universidad, que, para generar un mayor impulso, desde el 2012 se implementaron estrategias para enviar a estudiantes al extranjero como su estancia de investigación. De esta manera el VIC se diversificaría en verano estatal (universitarios que realizan su estancia de investigación en el Estado de residencia), nacional e internacional. La disminución de participantes en el 2018 y 2019 pudo deberse a que se estableció la titulación por las modalidades de tesis o artículo científico publicado como requisito para participar en el programa. Debido a que el año 2020 ha sido atípico, la institución no consideró pertinente abrir la convocatoria para realizar estancias nacionales e internacionales, sin embargo, se habilitó el Verano de la Investigación Científica Virtual, con una duración de ocho semanas con profesoras y profesores de la misma Institución, quienes fungen en la plataforma como responsables de proyectos de investigación registrados en la 
base de datos de la institución. Dadas las circunstancias, solo se presentarán estadísticas del número de participantes, del profesorado como del estudiantado.

Figura 1

Número de alumnado veraneante ${ }^{2}$ por año

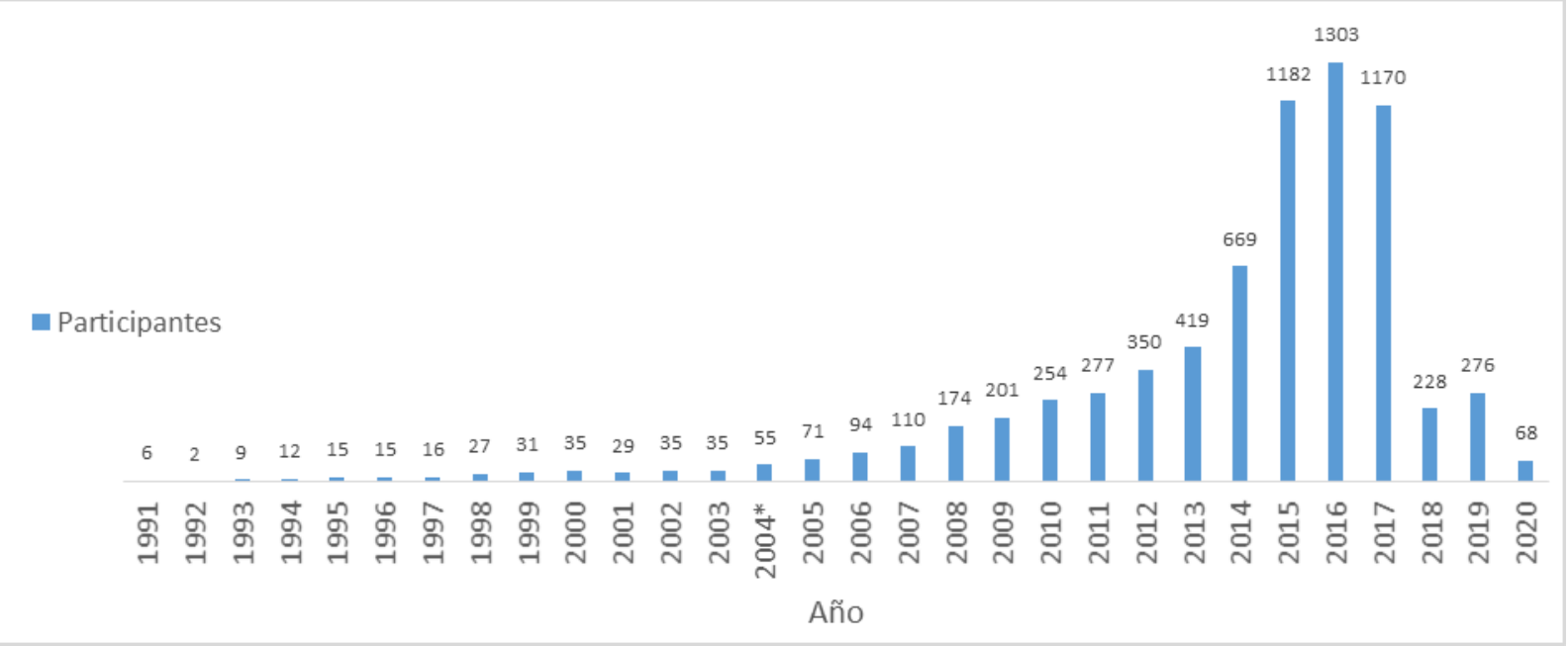

Fuente: Elaboración propia con información de la Secretaría de Investigación, Posgrado y Vinculación de la UJAT, 2020.

EI VIC de la UJAT ha alineado su reglamento y propósito a los de la AMC. Su objetivo primordial es:

fomentar el interés de los alumnos de licenciatura por la actividad científica, en las diferentes áreas de conocimiento, mediante la realización de una estancia de investigación durante 7 o 9 semanas en las más prestigiosas instituciones y centros de investigación del país o el extranjero. (UJAT, 2018, p. 2)

La apertura de la convocatoria se da el primer trimestre de año, se mantiene aproximadamente dos meses para que los postulantes reúnan los requisitos establecidos. Desde su creación hasta el 2016, debían contar con un promedio mínimo de 8.0, y un avance curricular del $50 \%$ al momento de su registro. Debido a la demanda eponencial, los requisitos se modificaron a partir del 2017. En la actualidad, el estudiantado debe contar con un promedio general de 9.0 y el avance del 65\% así como contar con la carta de aceptación del profesorado con quien desea realizar su estancia. Actualmente, la plataforma permite la interacción eficaz entre la institución, el profesorado y el alumnado.

\footnotetext{
${ }^{2}$ Estudiante de licenciatura que realizó su estancia de investigación a nivel estatal, nacional o internacional bajo el programa del Verano de la Investigación Científica.
} 
Al finalizar la estancia, debe emitir una carta de liberación del VIC, y contestar la encuesta que recaba la evaluación y comentarios generales acerca del alumnado veraneante. El alumnado veraneante contesta dos evaluaciones, la primera, a priori, denominada pre-verano, para conocer sus expectativas, la segunda, pos-verano para conocer sus impresiones e identificar si sus expectativas se cumplieron.

La aceptación del VIC de esta Universidad ha sido exponencial situándola como una de las instituciones con mayor participación en el programa a nivel nacional (Magaña, Aguilar, Pérez, Quijano y Argüelles, 2014). Como puede apreciarse en la concurrencia del alumnado veraneante, así como del profesorado en el período del 2013-2017. Esto no fue fortuito, se debió a implementacón de estrategias de difusión, a las visitas in situ para aclarar dudas, al uso de las redes sociales para mayor cobertura, por último, la creación de una plataforma electrónica que permite por un lado, monitorear la interacción directa y asíncrona de la administración-profesorado-estudiantado; por el otro lado, evaluar la conducta de los becarios en las estancias han contribuido al aumento de participación del profesorado a nivel nacional e internacional (ver Tabla 1 y Tabla 2).

Tabla 1

Profesorado participante según tipo de estancia en el VIC del 2016-2020

\begin{tabular}{lcccc}
\hline Año & Total de Profesorado & Estatal & Nacional & Internacional \\
\hline 2016 & 716 & 92 & 604 & 20 \\
2017 & 535 & 91 & 428 & 16 \\
2018 & 132 & 27 & 104 & 1 \\
2019 & 165 & 52 & 108 & 5 \\
2020 & 34 & 33 & 1 & 0 \\
\hline
\end{tabular}

Fuente: Elaboración propia con información de la Secretaría de Investigación, Posgrado y Vinculación de la UJAT, 2020.

Tabla 2

Estudiantado participante según tipo de estancia en el VIC del 2016-2020

\begin{tabular}{lcccc}
\hline Año & Estudiantado & Estatal & Nacional & Internacional \\
\hline 2016 & 1296 & 152 & 1112 & 32 \\
2017 & 1179 & 185 & 974 & 20 \\
2018 & 227 & 54 & 172 & 1 \\
2019 & 276 & 80 & 188 & 8 \\
2020 & 68 & 67 & 1 & 0 \\
\hline
\end{tabular}

Fuente: Elaboración propia con información de la Secretaría de Investigación, Posgrado y Vinculación de la UJAT, 2020.

Como la interacción profesorado científico- alumnado en formación detona este programa, es por ello que el propósito de este estudio es identificar la percepción que este binomio tienen sobre su rol en la formación científica y a partir de ahí, proponer las estrategias y mecanismos que enriquezcan el programa del VIC. 


\section{Fundamentación teórica}

En el campo de la investigación existen conceptos ligados dada su finalidad, tal es el caso de la información, la investigación científica y la formación científica. Dichos conceptos asociados a las acciones generadas a partir de las funciones del profesorado investigador. Para Peña (1995) la información científica ofrece una manera diferente de pensar, que obliga en cierta forma a los individuos a rechazar aquello que no se fundamente en hechos o razonamientos claros.

La investigación es una actividad sustantiva que no puede desentenderse del compromiso de generar interés e involucramiento de las nuevas generaciones, la juventud encontrará una experiencia invaluable como coadyuvante en su vocación científica, ampliando sus conocimientos y sus opciones para futuras etapas en su formación profesional, como menciona Restrepo (2003, p. 196) "la investigación puede ser vista desde la pedagogía y desde el ejercicio misional de generar conocimiento, propio de la educación superior".

Para Peña (2015) la investigación es la herramienta que nos coloca cara a cara con la realidad. Además, es un estímulo para resolver las dudas a las que nos enfrentamos, los problemas que agobian al ser humano, a las sociedades.

La formación científica parte del supuesto de enseñar a analizar los hechos a partir del acto mismo, buscar sus causas, explicarlas de manera metodológica y generar nuevas ideas, para reutilizarlas posteriormente.

Las instituciones deben apostar por la formación científica desde las aulas, es un hecho que carecer de ella, pone en desventaja a quienes se inician en la investigación, "la relación con el conocimiento que debe existir en todos los procesos académicos, tanto en el aprendizaje, por parte de los alumnos y alumnas, como en la renovación de la práctica pedagógica por parte del equipo de docentes" (Restrepo, 2002, p. 7).

Como afirman Rojas y Méndez (2017, p. 55):

En el campo de la educación en estudio de la actitud hacia la ciencia en general y hacia la investigación en particular, está íntimamente relacionada con la existencia de una formación significativa, representada en el desarrollo de las capacidades de profesores y estudiantes de elaborar y disponer del conocimiento de una manera dinámica, provocadora y concurrente con los propósitos de una educación integral. 
Por su parte, la investigación científica y el desarrollo tecnológico son los motores para aumentar la productividad y el crecimiento económico de un país (Undurraga, 2013). En este contexto es donde entra la función de las universidades, los centros de investigación y todas aquellas estancias u organismos que promueven y generan investigación científica.

El estudio de la formación investigativa es un tema ya abordado desde diferentes perspectivas, no obstante la importancia de este trabajo estriba en la relación del papel formador del profesorado científico, como un agente de enseñanza y motivación en un programa institucional como el Verano de la Investigación Científica, que pretende involucrar a postulantes en actividades investigativas, tema de interés en el contexto de la investigación debido al estancamiento de generación de conocimiento útil aplicable a la sociedad.

\subsection{El rol del profesorado investigador en la formación científica}

La idea de formar es una cuestión relativamente nueva en la historia de la educación (Rojas, 2009), y es posible que este fenómeno haya tomado con relativa sorpresa a las Instituciones de Educación Superior (IES), situación por la cual no se cuenta con un programa en el currículo funcional en esta tarea sustantiva, razón por la cual la mayoría de los participantes no estén dispuestos a continuar con su formación científica incluso, la falta de preparación permea desde los profesores hasta el estudiantado pero, sí se espera que las universidades, formadoras de científicos, dispongan de mejores elementos internos y externos, pedagógicos y didácticos, para incentivar la formación científica (Rojas, Méndez y Rodríguez, 2012).

El rol del profesorado como agente motivante debe contar con características y funciones que coadqyuven en su tarea de dirigir al estudiantado en su formación científica. Como sostiene Aranguren (2007, p. 179):

Es un sujeto en formación permanente que explora los acontecimientos que se suscitan en el aula de clase a objeto de elaborar diagnósticos, seleccionando, planificando y organizando acciones acordes con las circunstancias. Propone proyectos de investigación e intervención, a fin de construir soluciones teóricas y prácticas en compañía con otros docentes. Hace uso de la práctica para adquirir el saber; desde las situaciones cotidianas construye nuevos conocimientos mediante la interpretación y comprensión de los contenidos implícitos de una práctica determinada. 
El profesorado investigador tiene funciones y componentes encaminados a fortalecer la motivación del estudiantado, a estimular su pensamiento crítico encaminado a generar más información que una vez procesado genere nuevo conocimiento. Estas funciones pueden resultar complejas para el profesorado debido a las actividades inherentes como miembro de una planta docente en una institución. Silvio (2000 p. 24) señala que el rol del profesorado incluye no sólo motivar al estudiantado, si no además cumplir con un determinado de cursos, contar con proyectos de investigación minimamnete financiados entre otras actividades relevantes.

Magaña, Surdez y Zetina (2012), señalan que el rol que desempeñamos está influenciado por la posición en la cual interactuamos. En este sentido, las funciones del profesorado están asociadas a una actividad educativa de formación científica y jerárquicamente privilegiado en la pirámide institucional, que el VIC está integrado por la administración, el profesorado científico y el alumnado veraneante como se ha mencionado anterioridad.

\section{Método}

\subsection{Enfoque}

Se realizó un análisis cuantitativo descriptivo, con la encuesta aplicada al alumnado participante de las 12 divisiones académicas que participaron en el programa del Verano de la Investigación Científica en el periodo 2016-2020, y al profesorado científico, a quienes por su ubicación geográfico, se les clasificó como estatales para quienes su estado de residencia es Tabasco, nacionales, quienes viven en otro Estado de México y los internacionales residentes en el extranjero que intervienen en su formación científica desde 2016 hasta 2020.

\subsection{Unidades de análisis}

Se realizó un censo del programa de VIC en el período 2016-2020, se consideró como grupo de estudio a dos tipos de participantes, al profesorado investigador de universidades, centros de investigación y hospitales y al alumnado veraneante de todas las licenciaturas, que realizaron su estancia de investigación a nivel estatal, nacional e internacional. Se tuvo consentimiento por parte de la Administración institucional para usar los datos estadísticos del programa institucional por lo que se consideró cada registro pero se resguardó su anonimato. 


\subsection{Técnicas de recolección}

Para efectos de evaluación interna de programa, se implementaron dos evaluaciones, denominadas como pre-verano, y pos-verano. La primera, la responde el veraneante, previo a su estancia, para conocer sus expectativas; la segunda, la cual es objetivo de este análisis, la responden tanto el profesorado como el alumnado. El instrumento de recolección de datos en ambos casos, fue la encuesta, dispuesta en línea, en la plataforma del VIC. Para el caso del profesorado, consta de nueve preguntas en total, que califica al alumnado en una escala del 0 al 10, con las variables de Asistencia, Puntualidad, Compromiso, Conocimiento, Integración, Iniciativa, Disposición y Tareas realizadas (ver Anexo).

La encuesta pos-verano aplicada al alumnado, dispuesta en línea, en la plataforma de VIC consta de seis preguntas que evaluaban la Experiencia y el rol del profesorado en una escala del 0 al 10. Con las variables Estancia, Apoyo, Expectativas, Guía, Actividades. Las preguntas de Estancia y Expectativas se orientaron a medir el grado de satisfacción y del logro de las expectativas; la pregunta de Apoyo para considerarlo como argumento para incrementar el monto del financiamiento; la pregunta de Guía para identificar quienes intervienen en el proceso de formación y por último la pregunta Actividades para conocer la congruencia de estancia-actividad (ver Anexo).

Cabe mencionar que estos instrumentos fueron elaborados por el Departamento de Promoción y Desarrollo de la Investigación de la Secretaría de Investigación, Posgrado y Vinculación, por ende, están establecidos a nivel institucional para su operatividad.

\subsection{Procesamiento de análisis}

La recolección de datos se hizo mediante la implementación de una encuesta en línea que se ubicó en la plataforma del VIC.

Una vez que pasó el período de la estancia, se creó una base de datos general en una hoja de cálculo en Excel donde se recabaron 4525 cuestionarios contestados en el periodo de 2016 al 2020. Se dividieron las encuestas por tipo de participante, 1581 para el profesorado y 3047 del alumnado. Una vez organizados, se procedió a realizar el análisis de los resultados de las doce divisiones académicas e identificar aquellos que se destacaban por su representatividad. Asimismo, se hizo una revisión de la literatura existente relacionada con la formación temprana en investigación en el estudiantado de pre-grado 3 .

\footnotetext{
${ }^{3}$ Licenciatura
} 


\section{Resultados}

Los resultados expuestos son por tipo de participante, en primera instancia el resultado relacionado con el profesorado, posteriormente los resultados de la encuesta aplicada al alumnado veraneante. La tabla 3 muestra también que en ambos casos, la participación de la mujer en los escenarios de la ciencia es cada vez mayor.

Tabla 3

Profesorado y estudiantado participante por género en el VIC 2016-2020

\begin{tabular}{lrrrr}
\hline Año & \multicolumn{2}{c}{ Profesorado } & \multicolumn{2}{c}{ Estudiantado } \\
& Hombres & Mujeres & Hombres & Mujeres \\
\hline 2016 & 355 & 360 & 530 & 766 \\
2017 & 325 & 210 & 447 & 732 \\
2018 & 73 & 59 & 85 & 142 \\
2019 & 83 & 82 & 106 & 170 \\
2020 & 15 & 19 & 26 & 42 \\
Totales & 851 & 730 & 1194 & 1852 \\
\hline
\end{tabular}

Fuente: Elaboración propia con información de la Secretaría de Investigación, Posgrado y Vinculación de la UJAT, 2020.

\subsection{Resultados de la encuesta al profesorado}

La Tabla 4 muestra el resultado de la encuesta aplicada al profesorado científico, con relación a las categorías de asistencia, puntualidad, compromiso, integración, iniciativa, disposición y cumplimiento de las tareas. Se identifica que el alumnado veraneante obtuvo un mejor promedio en los rubros de Asistencia y entrega de tareas. En el campo de Conocimiento obtuvieron el promedio más bajo.

Tabla 4

Promedio de alumnado veraneante por categoría en el VIC 2016-2020

\begin{tabular}{lc}
\hline Categoría & Promedio \\
\hline Asistencia & 9.6 \\
Puntualidad & 9.5 \\
Compromiso & 9.5 \\
Conocimiento & 9.3 \\
Integración & 9.4 \\
Iniciativa & 9.5 \\
Disposición & 9.5 \\
Tareas & 9.6 \\
\hline
\end{tabular}

Fuente: Elaboración propia con información de la Secretaría de Investigación, Posgrado y Vinculación de la UJAT, 2020. 
Las Divisiones Académicas con mayor puntaje general fueron: División Académica de Ciencias Económico Administrativas (DACEA), División Académica de Informática y Sistemas (DAIS), División Académica Multidisciplinaria de Comalcalco (DAMC) y División Académica Multidisciplinaria de Jalpa de Méndez (DAMJM). Se pudo obervar una estrecha relación entre los rubros de Asistencia y cumplimiento de Tareas. A mayor asistencia más tareas entregadas (ver Anexo).

La Disposición de obtener nuevos aprendizajes, puede identificarse en las respuestas vertidas del profesorado científico, por ejemplo:

S2. "Realmente me siento muy satisfecho con el trabajo que ha realizado este alumno dentro de la dirección de mi disciplina, tiene grandes valores y disposición por aprender, ojalá tuviéramos muchos alumnos con él. Pienso que el programa debe ser muy exitoso con alumnos como este".

Con respecto a la entrega de Tareas, se identificó que hubo actitudes negativas y positivas del estudiantado y en este sentido, el profesorado recomienda establecer un lineamiento más riguroso para seleccionar a quienes participen en el programa.

S2. "En general el estudiante mostró una actitud positiva a todas las tareas asignadas. Durante su estancia el alumno demostró ser una persona con un alto sentido de responsabilidad y ética profesional. Entre sus principales cualidades destacan su gran iniciativa para desarrollar tareas asignadas, capacidad de comunicación, facilidad para desempeñar y apoyar trabajos en equipo".

Ramos y Sotomayor (2008) señalan que las deficiencias metodológicas percibidas por estudiantes en su proceso de formación, constituye una de las razones para no realizar investigación. Razón que da sentido a que el campo Conocimiento fue el ítem con el promedio general más bajo, de 9.3. Esto significó que a pesar de que el alumnado veraneante tiene la disposición de aprender, les falta conocimiento en la disciplina. Este resultado se apoya con los siguientes comentarios:

S3. "Incrementar el nivel de lectura de los alumnos, y a la búsqueda de información".

Por último, es importante hacer del conocimiento que la pregunta de Comentarios generales fue abierta, para que el profesorado pudiese verter sus impresiones generales, y en función de éstos, se pudo recabar la información que solventara las preguntas anteriores. 


\subsection{Resultado de la encuesta hecha al estudiantado veraneante en pos-verano}

Se relacionaron las preguntas con un identificador, quedando las categorías de Estancia, Apoyo, Expectativas, Guía y Actividades realizadas. Se identificó que tanto la Estancia y las Expectativas obtuvieron una calificación alta, y el rubro de Apoyo económico fue el de menor puntaje como puede verse en la Tabla 5.

Tabla 5

Promedio de la encuesta pos-verano según categorías en el VIC 2016-2020

\begin{tabular}{lc}
\hline Categoría & Promedio \\
\hline Estancia & 9.2 \\
Apoyo & 7.1 \\
Expectativas & 8.9
\end{tabular}

Fuente: Elaboración propia con información de la Secretaría de Investigación, Posgrado y Vinculación de la UJAT, 2020.

En relación a los resultados de pregunta de Expectativas, se observaron reacciones en ambos extremos. Por un lado, se encuentra la siguiente:

S1. "Deben considerar que existen investigadores que no cumplen con las expectativas $y$ no deben ser ofertados, es una mala experiencia tener que trabajar de mala forma con ellos". Las respuestas pueden ser el reflejo de la inexperiencia o desinterés de este profesorado en la formación científica del alumnado, que a su vez pueden ser determinantes en la decisión de los veraneantes de mantenerse 0 no en la investigación.

Por otro lado, en el VIC 2020, hubo comentarios positivos con respecto a las Expectativas: S17. "Fue un gusto trabajar con el investigador, considero que aprendí mucho de él, de igual forma, fortaleció varias de las competencias y habilidades adquiridas en el transcurso de la licenciatura. Sin duda, a pesar de las adversidades causadas por la pandemia, se pudo trabajar de una forma eficiente y agradable gracias a la aplicación de Teams y a los esfuerzos de las personas a cargo del VIC. Sería un gusto repetir está experiencia en el futuro".

La propuesta de un VIC virtual resultó interesante como alternativa como los interesados. El profesorado y la población estudiantil están conscientes que no se adquiere lo esperado de una estancia académica convencional pero resulta una alternativa viable para 
las actividades de formación científica. Como afirman Rojas y Méndez (2017) la actitud sobre la investigación en los estudiantes es un indicador sobre la calidad de la educación, y la importancia que tiene para el estudiante acercarse a una formación más cercana al campo del desarrollo científico (p. 55).

La universidad hizo una distribución de los apoyos según el tipo de estancia que se realizó. A quienes realizaron un verano estatal se les otorgó cinco mil pesos, para quienes realizaron una estancia en otro Estado, diez mil, y quienes viajaron al extranjero se les otorgó un apoyo por veinte mil pesos. La calificación general en este rubro fue la más baja con 7.1, porque de acuerdo a las impresiones vertidas, tales montos resultaron insuficientes.

S3. "El tema de estudio fue interesante, se me hizo aceptable la experiencia por primera y única vez, pero en lo personal se debería aumentar el apoyo económico que brinda la UJAT puesto que la cantidad asignada no cubre en su totalidad los gastos de renta, transporte y personales principalmente".

La Figura 2 muestra quién fue el Guía del estudiantado durante su estancia, se identificaron a personas becarias de posgrado y personas técnico-académicas aparte del propio profesorado. Para la institución es de interés tener claro si esta actividad se queda asignada a otra persona integrante en el proceso de formación.

Figura 2

Guía responsable del alumnado veraneante en estancia del VIC del 2016-2020

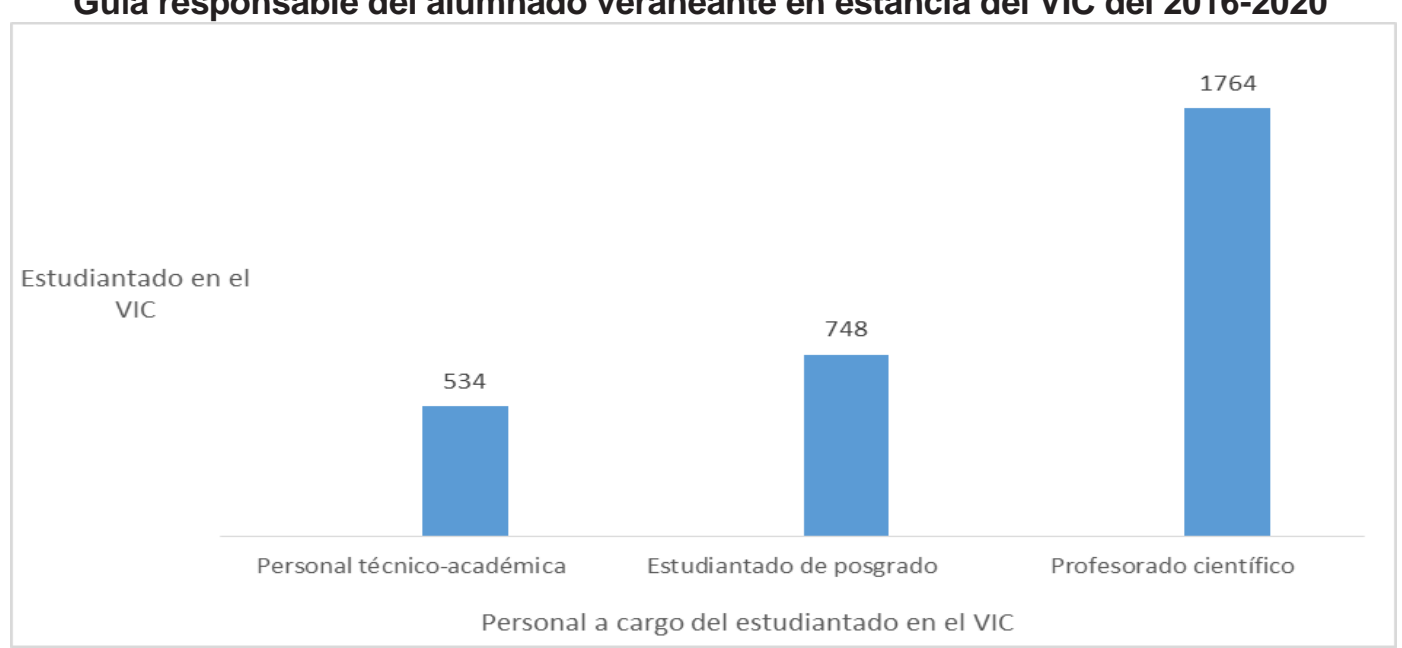

Fuente: Elaboración propia con información de la Secretaría de Investigación, Posgrado y Vinculación de la UJAT, 2020. 
En el periodo evaluado, el $42 \%$ de la población no fue atendida por el profesorado científico debido a que tenía diversas actividades y compromisos, sin embargo, no demostraron inconformidad, todo lo contrario, consideraron que su experiencia fue gratificante, por ejemplo:

S1. "Fue un excelente verano científico, en el cual pude descubrir y aprender cosas nuevas en el área de la investigación científica, gracias a la ayuda del Dr. Palomo y los catedráticos que nos apoyaron con la investigación que realizamos, pude entregar un excelente trabajo. Sin duda alguna volvería a participar en otro verano y así seguir aprendiendo y reforzando mis conocimientos".

Se pudo identificar el tipo de actividades que los veraneantes realizaban en su estancia de investigación, como puede observarse en la Figura 3, el 53\%, se concentró en desarrollar un proyecto o un artículo lo que es congruente con el objetivo de la estancia de investigación.

Figura 3

Actividades del alumnado veraneante en su estancia del VIC del 2016-2020

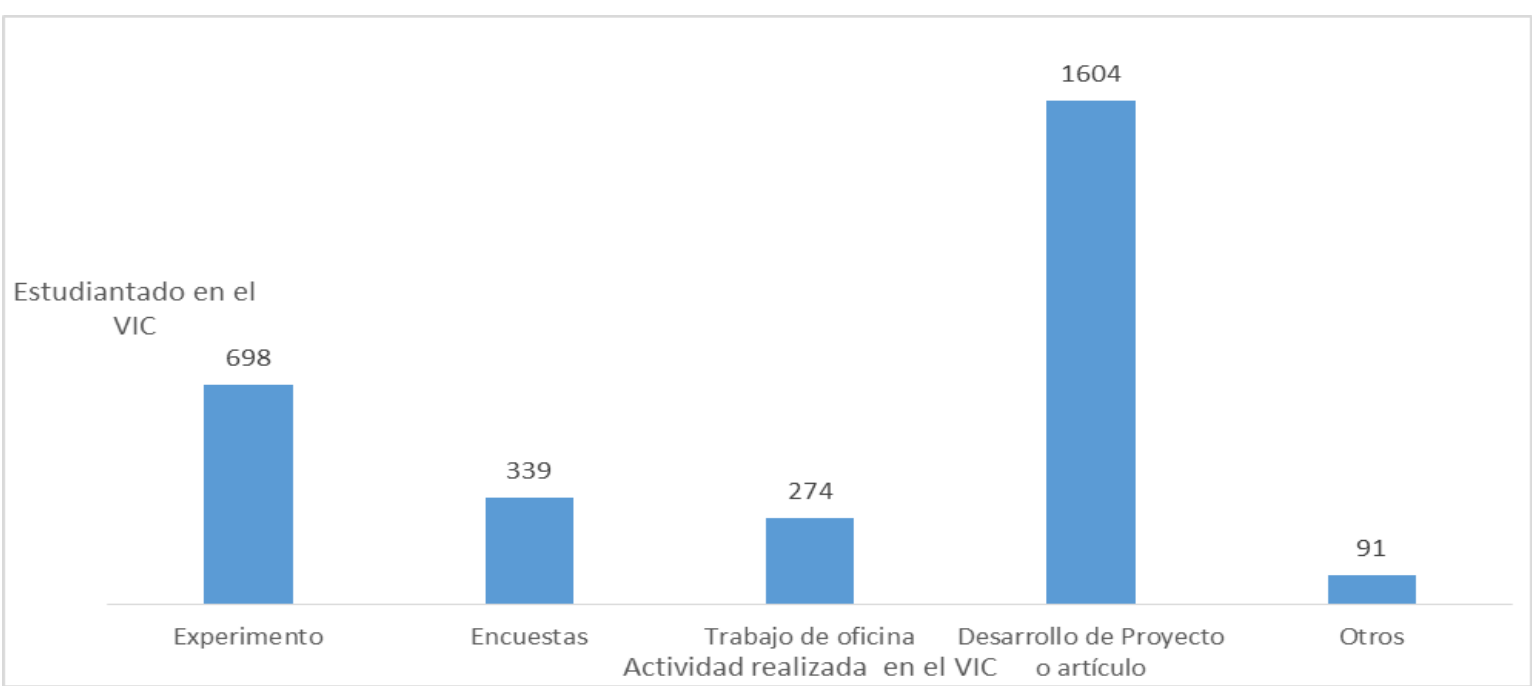

Fuente: Elaboración propia con información de la Secretaría de Investigación, Posgrado y Vinculación de la UJAT, 2020.

Como pudo observarse, las actividades fueron variadas, dependiendo el área, la disciplina y el proyecto en el cual colaboró el alumnado. La mayoría mostró satisfacción porque el profesorado científico los introdujo en tareas que desconocían, en el caso de quienes que trabajaron en laboratorios, tuvieron que leer manuales y tomar cursos de inducción previo al uso de equipos. 
S4. "La estancia fue muy buena y la doctora-investigadora con la que trabajamos siempre estuvo al pendiente de nosotros, realizamos de todo, encuestas, trabajo de oficina, trabajo en campo (centro de salud) y desarrollo del proyecto con la doctora, fue muy buena mi estancia, realmente me siento muy satisfecho".

Cabe hacer mención que las opiniones compartidas de ambos participantes fueron hechas en una pregunta abierta, fueron de utilidad para plasmar sus opiniones generales, inconformidades, felicitaciones y sugerencias y en función de ello mejorar las políticas del programa del VIC, así como el rol del profesorado científico como agente de cambio.

\section{Discusión y conclusiones}

El trabajo formativo que el profesorado realiza con estudiantes en su estancia puede ser determinante se reflejó en los resultados expuestos con anterioridad, el $76 \%$ de la población estudiantil realizó actividades inherentes a la investigación científica. ya que como señala Rojas (2009), enseñar a investigar constituye un elemento transversal en la organización de los procesos de formación universitaria, por lo menos desde la declaración formal y generalizada de los proyectos educativos de las universidades.

Con base en ello, la población estudiantil señaló que el profesorado científico es trascedente tanto en su formación científica como para su aprendizaje extra-aula, técnicas y estrategias que los induzcan a estudiar un posgrado; tendría que verse como una aventura que lleve a una mejora social y no simplemente como una actividad elitista. En este sentido, debe contar con capacidad de trabajo colaborativo y autocrítico para identificar y mejorar sus vacíos académicos y humanísticos en pro de la formación a generaciones sucedáneas, será de interés institucional generar un perfil idóneo de profesorado científico, basado en cuatro aspectos capaces de transmitir: actitud, conocimiento, habilidades y ética que delineen al estudiante en la función sustantiva de investigar.

De acuerdo a los resultados del periodo estudiado, el $60 \%$ de participantes en el VIC fueron mujeres. Significa que el género femenino cada vez ocupa un rol más determinante en la ciencia, si se considera que apenas en el s. XX la mujer tenía otro rol en la sociedad. La carrera científica en las sociedades era asumida como una actividad impropia para la mujer (Sanabria, 2018, p. 189). El rol de la mujer en la ciencia no es el objetivo de este estudio ni se trata de señalar las brechas de género, es visible que la mujer científica está 
marcando líneas que motivan a las congéneres a no desistir en el campo; futuros estudios en la materia darán más elementos para sostener esta premisa.

El programa institucional del Verano de la Investigación Científica es estratégico para las universidades ya que en esta estancia se desarrollan las habilidades, competencias y conocimiento, como Barbier (1999) señala que se trata de dinamizar el proceso de transformación de la persona en términos de la evolución de sus potencialidades y transformación de capacidades. Como pudo verse en los resultados, el nivel de satisfacción del alumnado y profesorado es buena, aunque se hayan presentado situaciones indeseables con una tasa de incidencia muy baja, pero han servido para generar más y mejores mecanismos de control.

Se aportan una serie de propuestas que tanto el profesorado científico como el alumnado exponen en sus comentarios generales. Entre ellos:

- Proponer una guía al profesorado participante y a la administración para darle un seguimiento oportuno y cercano al proceso.

- Mejorar la selección del profesorado científico que participará en el programa a nivel estatal, como pudo observarse, la expectativa hacia la investigación se ve mermada a consecuencia de la apreciacón negativa por parte del alumnado.

- Fortalecer los foros de divulgación existentes que son escenarios en el que la población estudiantil participante comparte sus experiencias y esto genera interés en otros.

- Profundizar en conocimientos de las materias académicas que pertenecen a las áreas básicas y tengan nociones del método científico, previo a la realización del programa de verano, para que lleguen con competencias investigativas puedan aprovechar mejor su estancia.

- Mejorar las encuestas de pre-verano, pos-verano y del profesorado científico para que las preguntas arrojen información sustancial del proceso y posteriormente hacer una investigación que permita obtener información significativa.

- Crear un programa transversal permanente en el que se capacite al alumnado veraneante en herramientas y habilidades investigativas, con el fin de mantener su interés en la investigación, y que a su vez, permita el incremento de titulación por la modalidad de tesis o publicación de artículo científico. 
- Establecer el VIC virtual como alternativa para el estudiantado que no alcance una beca, o que no cuente con la posibilidad de realizar la estancia presencial en otra entidad.

Para el caso específico de la UJAT, es importante prestar atención a las propuestas ya que en concordancia con Rodríguez (2015, p. 92), “la formación universitaria presentará cada vez más un escenario múltiple y diverso en el que será muy difícil aferrarse a viejos planteamientos".

De manera reiterada, queda claro que el rol que juega el profesorado científico trasciende en la investigación per se, y en la formación científica del alumnado veraneante; razón por la que las instituciones de educación superior la consideran entre sus actividades sustantivas y en particular la UJAT, ha hecho una inversión económica sustancial en el VIC, como un semillero de nuevas personas científicas, desde su institucionalización hasta el 2017, año en el que este programa tuvo su mayor auge. La disminución considerable en la participación que se observó en el 2018 y 2019 se debió a diversos factores, sobre todo la disponibilidad financiera institucional, misma que llevó a incrementar los requisitos de acceso. En el 2020, pese a las circunstacias, el estudiantado pudo realizar su estancia en la modalidad virtual ante las medidas de distanciamiento social. Significó un compromiso por parte de la institución, del profesorado y de la población estudiantil.

La reflexión sobre la formación temprana en investigación será necesaria en estudios prospectivos porque las futuras investigaciones y exploraciones deberán perfilar al profesorado y alumnado hacia el futuro, prepararlos para las problemáticas que resolverán desde su disciplina. Al final, serán responsables de formarse para responder a las problemáticas contextuales, pensando en las problemáticas globales pero actuando local, tal como la pandemia actual que nos traza directrices a las que debemos adaptarnos en un corto tiempo y sobre la cual deberán realizarse futuros estudios.

\section{Agradecimientos}

A la Secretaría de Investigación, Posgrado y Vinculación de México, en particular a la Lic. Bellanira Pérez Hernández del Depto. De Promoción y Desarrollo de Investigación por ser generosos con la información. 


\section{Referencias}

Academia Mexicana de Ciencias (AMC). (2020). Academia Mexicana de Ciencias, A.C. Recuperado de https://www.amc.edu.mx/amc/images/verano/CONVOCATORIAXXXVerano-2020L.pdf

Aranguren Peraza, Gilberto. (2007). La investigación-acción sistematizadora como estrategia de intervención y formación del docente en su rol de investigador. Revista de Pedagogía, 28(82), 173-195. Recuperado de https://www.redalyc.org/pdf/659/65908202.pdf

Barbier, Jean Marie. (1999). Prácticas de formación. Evaluación y análisis (Formación de formadores). Argentina: Ediciones Novedades Educativas.

Magaña Medina, Deneb Elí., Surdez Pérez, Edith Georgina. y Zetina Pérez, Carlos David. (2012). Ambigüedad de rol en investigadores. Revista Internacional Administración \& Finanzas, 5(3). Recuperado de http://www.theibfr2.com/RePEc/ibf/riafin/riaf-v5n32012/RIAF-V5N3-2012-5.pdf

Magaña Medina, Deneb Elí., Aguilar Morales, Norma., Pérez Cano, Marina., Quijano García, Román Alberto. y Argüelles Ma, Luis Alfredo. (2014). Motivaciones y limitantes en la formación en investigación a través del programa de verano científico: Un estudio en una muestra de estudiantes universitarios. Revista Internacional de Administración y Finanzas, 7(6). Recuperado de ftp://ftp.repec.org/opt/ReDIF/RePEc/ibf/riafin/riaf-v7n62014/RIAF-V7N6-2014-8.pdf

Naranjo Pereira, María Luisa. (2009). Motivación: perspectivas teóricas y algunas consideraciones de su importancia en el ámbito educativo. Revista Educación, 33(2), 153-170. Doi 10.15517/revedu.v33i2.510

Palés, Jorge y Arcadi, Gual. (2004). Recursos educativos en ciencias de la salud. Educación Médica, $7(1)$. Recuperado de http://scielo.isciii.es/scielo.php?script=sci arttext\&pid=S1575-18132004000200002

Peña, Antonio. (1995). La investigación científica en México. Estado actual, algunos problemas y perspectivas. Perfiles Educativos, (67). Recuperado de https://www.redalyc.org/pdf/132/13206702.pdf

Peña Orozco, Carlos Alberto. (2015). La importancia de la investigación en la Universidad: Una Reinvindicación del Sapere Aude Kantiano. Revista Amauta, (25). Recuperado de https://dialnet.unirioja.es/descarga/articulo/5440965.pdf

Quintero Corzo, Josefina., Munévar Molina, Raúl Ancízar. y Munévar Quintero, Fabio Ignacio. (2008). Semilleros de investigación: Una estrategia para la formación de investigadores. Educación y Educadores, 11(1), 31-42. Recuperado de http://www.redalyc.org/articulo.oa?id=83411103

Ramos Rodríguez, Mariana. y Sotomayor, Renzo. (2008). Realizar o no una tesis: razones de estudiantes de medicina de una universidad pública y otros asociados. Rev Peru Med Exp Salud Pública, 25(3). Recuperado de https://pdfs.semanticscholar.org/b88d/be47e639c8cbda1 c41f78420bcd6c35f5e85.pdf? $\mathrm{ga}=2.2689592 .902491440 .1589510650-1762853561.1589510650$ 
Restrepo Gómez, Bernardo. (2002). Conceptos y aplicaciones de la Investigación Formativa, y criterios para evaluar la investigación científica en sentido estricto. Recuperado de https://www.epn.edu.ec/wp-content/uploads/2017/03/Investigaci\%C3\%B3n-FormativaColombia.pdf

Restrepo Gómez, Bernardo. (2003). Investigación formativa e investigación productiva de conocimiento en la universidad. Nómadas, (18), 195-202. Recuperado de http://www.redalyc.org/articulo.oa?id=105117890019

Rodríguez Espinar, Sebastián. (2015). Los estudiantes universitarios de hoy: Una visión multinivel. Revista de Docencia Universitaria, 13(2), 91-124. doi: https://doi.org/10.4995/redu.2015.5440

Rojas Betancur, Héctor Mauricio. (2009). Formar investigadores e investigadoras en la universidad: Optimismo e indiferencia juvenil en temas científicos. Revista Latinoamericana de Ciencias Sociales, Niñez y Juventud, 7(2). Recuperado de http://dialnet.unirioja.es/servlet/articulo?codigo=3236679\&info=resumen\&idioma=ENG

Rojas Betancur, Héctor Mauricio., Méndez Villamizar, Raquel. y Rodríguez Prada, Ángela. (2012). Índice de actitud hacia la investigación en estudiantes del nivel de pregrado. Entramado, 8(2), 216-229. Recuperado de http://www.redalyc.org/pdf/2654/265425848014.pdf

Rojas Betancur, Héctor Mauricio. y Méndez Villamizar, Raquel. (2017). Procesos de formación en investigación en la Universidad: ¿Qué le queda a los estudiantes? Sophia, 13(2). Recuperado de http://revistas.ugca.edu.co/index.php/sophia/article/view/261/1264

Sanabria Rojas, Quira Alejandra. (2018). Rol de la mujer en la ciencia, su enseñanza en Colombia y diversidad cultural: aproximación desde la historia nacional: Universidad Distrital Francisco José de Caldas. Recuperado de https://cutt.ly/cflocYJ

Silvio Vacarezzca, Leonardo. (2000). Las estrategias de desempeño de la profesión académica. Ciencia periférica y sustentabilidad de rol de investigador universitario. Redes 7(15), 15-4. Recuperado de https://www.redalyc.org/pdf/907/90701501.pdf

Universidad Juárez Autónoma de Tabasco [UJAT]. (2004). Plan de Desarrollo Institucional 2004-2008. Dirección General de Planeación y Evaluación Institucional. Recuperado de http://www.archivos.ujat.mx/DGPEl/PDI\%202004-2008.pdf

Universidad Juárez Autónoma de Tabasco [UJAT]. (2016). Plan de Desarrollo Institucional 2016-2020 (Colección Justo Sierra, Documentos y Estudios sobre la Universidad). Recuperado de http://www.archivos.ujat.mx/2016/rectoria/PDI FINAL2020.pdf

Undurraga Pellegrini, Sofía. (2013). Evaluación del desarrollo de competencias de investigación en pregrado en la Escuela de Ingeniería Civil de la Pontificia Universidad Católica de Chile. (Tesis de Maestría). Pontificia Universidad Católica de Chile, Chile. Recuperado de https://repositorio.uc.cl./handle/11534/1838 


\section{Anexo}

\section{Índice de preguntas al profesorado}

\section{Pregunta}

¿El estudiante llegaba todos los días?

¿El estudiante llegaba a la hora acordada?

¿Realizaba las actividades asignadas?

¿Mostró conocimiento en el área?

¿Se integró fácilmente al equipo de trabajo?

¿Mostró iniciativa en las actividades encomendadas?

¿Mostró disposición para aprender nuevos métodos de

aprendizaje?

¿Entregaba las tareas asignadas?

Comentarios generales

\section{Índice de preguntas de pos-verano para el estudiantado}

\begin{tabular}{l}
\hline Pregunta \\
\hline ¿Qué te pareció la estancia del VIC? \\
¿Fue suficiente el apoyo económico? \\
¿Se cubrieron tus expectativas? \\
En caso de que el investigador no haya trabajado de manera directa \\
contigo, ¿quién te tutoró en tu estancia? \\
¿Qué tipo de actividad realizaste en tu estancia? \\
Comentarios generales \\
\hline
\end{tabular}

Promedio de las divisiones académicas por categoría en el VIC 2016-2020

\begin{tabular}{lcccccccccccc}
\hline & DACA & DACB & DACBIOL & DACS & DACEA & DACSYH & DAEA & DAIS & DAIA & DAMC & DAMMM & DAMRIOS \\
\hline Asistencia & 9.5 & 9.6 & 9.7 & 9.6 & 9.8 & 9.5 & 9.4 & 9.9 & 9.7 & 9.6 & 9.8 & 9.6 \\
Puntualidad & 9.5 & 9.4 & 9.3 & 9.3 & 9.6 & 9.2 & 9.3 & 9.8 & 9.5 & 9.5 & 9.7 & 9.5 \\
Compromiso & 9.6 & 9.6 & 9.7 & 9.6 & 9.8 & 8.2 & 9.4 & 9.9 & 9.6 & 9.6 & 9.6 & 9.6 \\
Conocimiento & 9.1 & 9.1 & 9.3 & 9.4 & 9.5 & 9.3 & 9.1 & 9.8 & 9.2 & 9.5 & 9.2 & 9.2 \\
Integración & 9.3 & 9.4 & 9.1 & 9.5 & 9.7 & 9.4 & 9.3 & 9.5 & 9.2 & 9.5 & 9.5 & 9.6 \\
Iniciativa & 9.4 & 9.5 & 9.5 & 9.6 & 9.7 & 9.1 & 9.4 & 9.7 & 9.5 & 9.7 & 9.5 & 9.6 \\
Disposición & 9.5 & 9.6 & 9.5 & 9.4 & 9.8 & 9.4 & 9.5 & 9.9 & 9 & 9.7 & 9.6 & 9.5 \\
Tareas & 9.6 & 9.6 & 9.6 & 9.6 & 9.9 & 9.0 & 9.5 & 10 & 9.6 & 9.7 & 9.6 & 9.5 \\
\hline
\end{tabular}


Revista indizada en

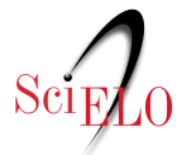

Distribuida en las bases de datos:

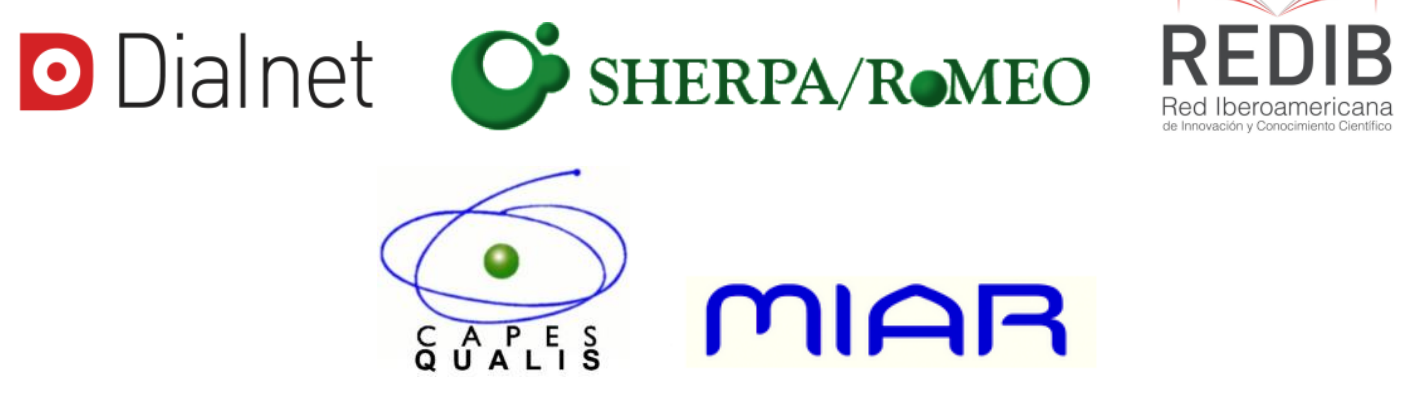

